\title{
Superação em Esquizofrenia: relato de casos
}

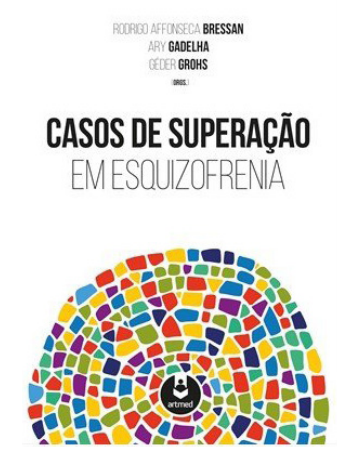

Palavras-chave

Esquizofrenia, recovery, estigma.

\section{Keywords}

Schizophrenia, recovery, stigma.

\author{
Overcoming Schizophrenia: case report \\ Mário César Rezende Andrade,2 \\ hitp://orid.org/0000-0003-2784-5209 \\ Bressan, RA; Gadelha, A; Grohs, G (orgs). Casos de superação em esquizofrenia. Porto Alegre: Artmed; 2017.
}

\section{RESUMO}

A esquizofrenia tem sido tradicionalmente vista como uma doença que leva a deterioração e incapacidade progressivas. Essa visão pessimista se faz presente na própria prática clínica em psiquiatria. O livro Casos de superação em esquizofrenia desafia tal visão, ao apresentar um número considerável de casos clínicos reais de pessoas com esquizofrenia que apresentaram um desfecho mais otimista. Cada capítulo apresenta um caso, descrito por seu clínico responsável e caracterizado por uma superação clínica e pessoal da doença. A obra contribui, de forma original, com o desenvolvimento de uma atitude mais positiva e de esperança em relação ao tratamento da esquizofrenia.

\section{ABSTRACT}

Schizophrenia has been traditionally viewed as a condition that leads to progressive impairment and disability. This pessimistic view is present in the clinical practice itself in psychiatry. The book Cases of recovery in schizophrenia challenges such a view by presenting a considerable number of real clinical cases of people with schizophrenia who presented a more optimistic outcome. Each chapter presents a case, described by its own clinician and characterized by a clinical and personal recovery of the condition. This work contributes, in an original way, to the development of a more positive and hopeful attitude towards the treatment of schizophrenia.

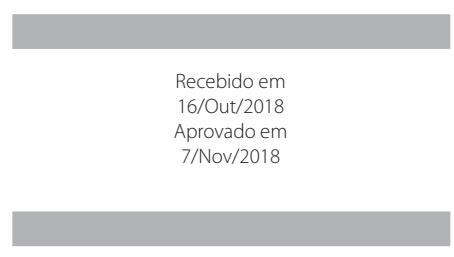

DOI: 10.1590/0047-2085000000227

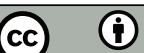

1 Universidade Federal de São Paulo (Unifesp), Departamento de Psiquiatria, Programa de Pós-Graduação em Psiquiatria e Psicologia Médica, São Paulo, SP, Brasil.

2 Universidade Federal de São João del-Rei (UFSJ), Departamento de Psicologia, São João del-Rei, MG, Brasil.

Endereço para correspondência: Mário César Rezende Andrade

Departamento de Psicologia, UFSJ, Campus Dom Bosco

Praça Dom Helvécio 74, sala 122

36301-160 - São João del-Rei, Minas Gerais, Brasil

E-mail:mariocesar@ufsj.edu.br 
A esquizofrenia é predominantemente representada como uma doença grave e incapacitante, que leva exclusivamente a um desfecho de deterioração progressiva em várias esferas da vida. Essa visão está presente na sociedade como um todo e até mesmo na própria psiquiatria, o que pode dificultar o tratamento e reforçar ainda mais tal visão negativa. Entretanto, nas últimas décadas, vários estudos têm mostrado que uma parcela significativa de pessoas com esquizofrenia pode atingir uma recuperação funcional e sintomatológica satisfatória, devido principalmente a progressos nas intervenções farmacológicas e psicossociais. Paralelo a essa maior possibilidade de recuperação clínica, tem sido proposto recentemente o conceito de superação (tradução usada no livro para o termo inglês "recovery"), que tem progressivamente orientado as políticas de saúde mental em diferentes países. Mais do que a melhora clínica, esse conceito está associado a um processo individual e contínuo de desenvolvimento de uma vida significativa, com esperança e com uma nova identidade além da doença, apesar das consequências negativas dela'.

Baseando-se nessas novas possibilidades, o livro Casos de superação em esquizofrenia ${ }^{2}$, lançado recentemente, desafia a visão pessimista predominante acerca da doença. O livro reúne 25 casos clínicos reais de pessoas diagnosticadas com esquizofrenia, escritos por alguns dos maiores psiquiatras brasileiros que atuam na área, responsáveis pelo atendimento a essas pessoas. Todos os casos têm em comum um desfecho mais otimista, caracterizado por um processo de superação, tanto clínico quanto pessoal. Cada caso é apresentado em um capítulo, sendo dividido nas seguintes seções: (1) identificação da pessoa; (2) motivo do atendimento com o clínico responsável pela descrição do caso; (3) história da pessoa, com ênfase no contexto de desenvolvimento da esquizofrenia, curso inicial dos sintomas e suas consequências; (4) achados do clínico, ou seja, como a pessoa se apresentou ao atendimento, as primeiras impressões e intervenções; (5) curso do caso, com os procedimentos e decisões que levaram à superação da doença, tanto em termos da melhora sintomatológica e funcional, quanto do desenvolvimento de uma identidade além da doença, com sentido e esperança; (6) discussão, na qual são citados os fatores decisivos para o processo de superação em cada caso.

Os casos do livro descrevem pessoas de diversas origens e classes, todos clinicamente graves ou moderados, apresentando desafios importantes para os clínicos, mas, mesmo assim, desenvolvendo a superação. Casos mais leves, quando bem tratados, possuem prognósticos ainda melhores. Apesar da diversidade e singularidade de cada pessoa, alguns fatores se destacam como decisivos para o processo de superação na maioria dos casos apresentados, entre eles: o estabelecimento da aliança terapêutica, o acerto colaborativo da medicação, o envolvimento dos familiares, o oferecimento de outras intervenções psicossociais e o apoio ao "empoderamento" da pessoa, na vida e no tratamento. A obra consiste, portanto, em uma iniciativa importante e original, que apresenta, por meio de casos reais, a possibilidade de superação da esquizofrenia. O livro pode também ser usado como um instrumento inovador de formação em saúde mental, preparando futuros clínicos para uma atitude mais realista e de esperança com relação à doença.

\section{AGRADECIMENTOS}

Este manuscrito foi redigido pelo autor durante seu estágio de pós-doutorado no Programa de Pós-Graduação em Psiquiatria e Psicologia Médica do Departamento de Psiquiatria da Universidade Federal de São Paulo (Unifesp), com bolsa e apoio da Coordenação de Aperfeiçoamento de Pessoal de Nível Superior - Brasil (Capes) - Código de Financiamento 001.

\section{REFERÊNCIAS}

1. Anthony WA. Recovery from mental illness: the guiding vision of the mental health system in the 1990s. Psychatr Rehabil J. 1993;16(4):11-23.

2. Bressan RA, Gadelha A, Grohs G (Orgs.). Casos de superação em esquizofrenia. Porto Alegre: Artmed; 2017. 